\title{
Synthesis of Water-Soluble Far-Red-Emitting Amphiphilic BODIPY Dyes
}

\author{
Kevin M. Bardon, ${ }^{\dagger}$ Scott Selfridge, ${ }^{*}{ }^{\dagger}$ Dany S. Adams, ${ }^{\dagger}$ Richard A. Minns, ${ }^{\dagger}$ Robert Pawle, ${ }^{\dagger}$ \\ Timothy C. Adams, ${ }^{\dagger}$ and Larry Takiff ${ }^{\dagger}$ \\ ${ }^{\dagger}$ Akita Innovations LLC, 267 Boston Road, Suite 11, North Billerica, Massachusetts 01862, United States \\ ${ }^{\ddagger}$ Tufts University, Tufts Center for Regenerative \& Developmental Biology, 200 Boston Avenue, Medford, Massachusetts 02155, \\ United States
}

\section{Supporting Information}

ABSTRACT: We report the synthesis of two water-soluble BODIPY dyes with far-red absorption and near-infrared fluorescence following cell membrane insertion. Introduction of dicationic or dianionic groups imparts water solubility and prevents translocation of the dye through the plasma membrane for highly effective labeling. The dicationic form is particularly well localized to the plasma membrane and
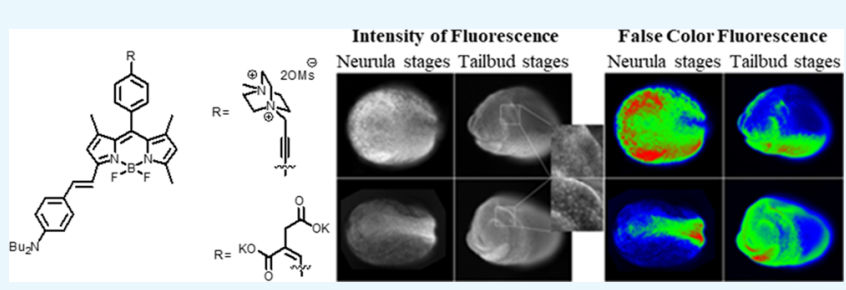
resists quenching even after $>8$ min of continuous light exposure. The dyes are almost completely nonemissive in water and other highly polar solvents, but display high-fluorescence yields in chloroform and upon insertion into the extracellular leaflet.

\section{INTRODUCTION}

Optical imaging of cells provides tremendous insight into a myriad of biological processes, ${ }^{1}$ and fluorescence-based imaging is an especially sensitive approach ${ }^{2}$ that is applied to wide-ranging scientific fields such as clinical diagnostics, ${ }^{3}$ biochemistry and molecular biology, ${ }^{4}$ and materials science. ${ }^{5}$ Fluorescence microscopy is well suited to cell-based investigations due to its inherent high sensitivity and specificity, spatiotemporal range and sampling capabilities, and biocompatability. ${ }^{6,7}$ Commonly used fluorescent reporters include proteins, inorganic compounds, and small molecule dyes. ${ }^{8}$ Of particular interest in bioimaging are far-red to NIR-emissive $(>650 \mathrm{~nm})$ dyes. The wavelength range of $650-1350 \mathrm{~nm}$ is commonly referred to as the "physiological" or "optical" window where light has its maximum depth of penetration into tissue. Additionally, NIR emitters show improved signal-tonoise ratio by avoiding autofluorescence resulting from indiscriminate excitation of various normal cellular components. ${ }^{9}$ Longer excitation wavelengths also reduce light scattering, and the potential for photoinduced cell damage as a high signal-to-noise ratio plus lower energy excitation allows much longer observations of viable cells. ${ }^{10}$

Notable NIR-emissive dyes include cyanines, ${ }^{11}$ squaraines, ${ }^{11}$ Alexa750, ${ }^{12}$ rhodamines, ${ }^{12}$ and phthalocyanines. ${ }^{13}$ These dyes show strong absorbance and fluorescence, but can suffer from a variety of performance issues, including aggregation, ${ }^{11}$ photobleaching, ${ }^{14}$ unspecific binding to cell components, ${ }^{15}$ and poor hydrophilicity. ${ }^{8,16}$ Dyes derived from 4,4-difluoro-4-bora$3 a, 4 a$-diaza-s-indacene ${ }^{17}$ (BODIPY dyes) possess similar photophysical properties (strong absorptivity and highfluorescence quantum yield) to the dyes listed above but are known to be photostable. ${ }^{18}$ As with other organic dyes,
BODIPYs can be synthetically modified to suit the target applications and several derivatives have been prepared that resist aggregation and have outstanding aqueous solubility. ${ }^{18,19}$ Improved aqueous solubility has facilitated their use in aqueous media and minimized cytotoxicity issues commonly seen when organic solvents such as ethanol, dimethylformamide, or dimethyl sulfoxide are required to predissolve and/or load dyes. ${ }^{20}$ Due to these desirable traits, preparation of BODIPY dyes is currently an active area of research in bioanalysis and bioimaging., ${ }^{9,21-24}$

\section{RESULTS AND DISCUSSION}

We have developed a novel BODIPY system, shown in Chart 1, that exhibits two interesting and valuable properties: extended residence time in cell membranes and a fluorescence "turn-on" effect in the presence of surfactant in aqueous solution. Both of these characteristics indicate applicability in cell membrane analyses. To demonstrate the potential use of these dyes in membrane analyses, we selected plasma membrane staining, an important application of fluorescencebased cell imaging. ${ }^{13}$ Outlining cells with fluorescent reporters provides critical spatial information. Cell membranes participate in numerous cellular events, including cell division and endocytosis. Furthermore, patency or disruption of the plasma membrane is a hallmark of apoptosis. ${ }^{25}$ Fluorescence-based cell membrane imaging allows for real-time visualization of these essential cellular events. ${ }^{26-28}$ In addition to membrane

Received: June 28, 2018

Accepted: October 1, 2018

Published: October 15, 2018 


\section{Chart 1. BODIPY Dyes 1 and $2^{a}$}

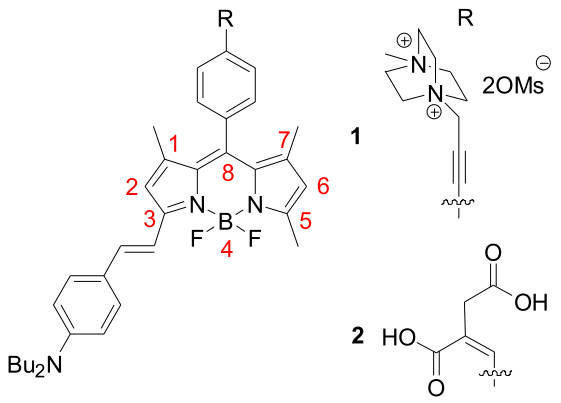

${ }^{a}$ Positions on the BODIPY core are labeled in red.

labeling, 1 shows additional utility as a voltage sensing dye, which will be the subject of an additional report.

In the effort to prepare a photostable, NIR fluorescent, water-soluble membrane probe, we designed dyes $\mathbf{1}$ and 2, shown in Chart 1. Both dyes are derivatives of borondipyrromethene (BODIPY), ${ }^{29,30}$ with a dibutylaminophenylene vinylene group attached to the 3-position on the BODIPY. ${ }^{31,32}$ This dibutylaminophenylene vinylene group extends the conjugation of the BODIPY chromophore, resulting in $>100 \mathrm{~nm}$ bathochromic shifts in absorbance and emission relative to the parent chromophore. ${ }^{5,33}$ We achieved water solubility by installing charged groups via phenylene linkers at the 8position. Dye 1 contains a dication prepared by alkylating diazabicyclo[2.2.2] octane (DABCO) and has excellent water solubility across the biological $\mathrm{pH}$ range. Dye 2 contains a dicarboxylic acid prepared with itaconic acid and is soluble in basic water. The butyl groups on the aminophenylene unit balance the oleophilic nature of one end of the molecule with the hydrophilic nature of the other end, resulting in an amphiphilic molecule able to partially insert into micelles or membranes with a hydrophilic end anchoring part of the molecule outside the membrane. We investigated other alkyl groups for the aniline moiety (dimethyl, dioctyl, methylhexadecyl) and the butyl groups provided the best oleophilic and hydrophilic combination. Both $\mathbf{1}$ and $\mathbf{2}$ label cell membranes, with the hydrophobic chromophore positioned on the membrane interior and the hydrophilic group extending into the extracellular area. In particular, 1 is an excellent label for cell plasma membranes with almost no signal visible from intracellular membranes observed over several hours.

We prepared dyes $\mathbf{1}$ and $\mathbf{2}$ following the procedure shown in Scheme 1, designed to prepare both dyes from a common intermediate. Intermediate $\mathbf{3}$ is an aminophenylene vinylenesubstituted BODIPY with an iodophenyl group in the meso position. We used the iodo group to add the charged groups on 1 and 2 . We prepared 3 by Knoevenagel coupling between commercially available 8-iodophenyl-1,3,5,7-tetramethyl BODIPY and 4-N,N-dibutylaminobenzaldehyde, using 0.5 equiv of aldehyde to suppress the formation of the bis-addition product. From 3, we prepared 2 in one step via Heck coupling with itaconic acid. We prepared intermediate 6 , the precursor to $\mathbf{1}$, by Sonogashira reaction between 3 and propargyl alcohol followed by mesylation of the alcohol group to give intermediate 6 . We transformed 6 into 1 by quaternization of 1-methyl-1-azonia-4-azabicyclo[2.2.2] octane mesylate, which was prepared by alkylation of $\mathrm{DABCO}$ by methyl methanesulfonate.
Scheme 1. Preparation of 1 and $2^{a}$

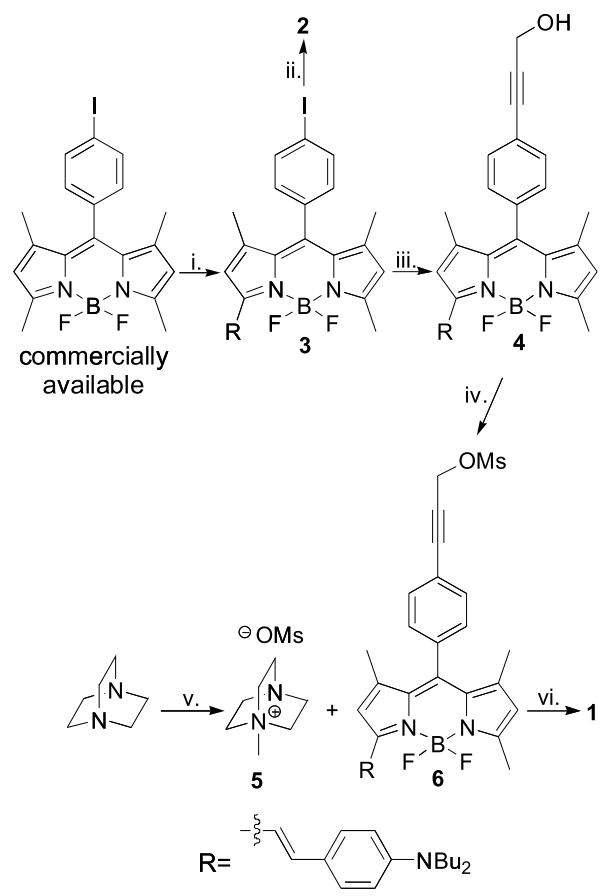

a (i) 4-N,N-Dibutylaminobenzaldehyde, $p$-toluenesulfonic acid monohydrate, piperidine, toluene. (ii) Itaconic acid, $\mathrm{Pd}(\mathrm{OAc})_{2}, \mathrm{P}(o \text {-tol })_{3}$, $\mathrm{NBu}_{4} \mathrm{Br}, \mathrm{MeCN}, \mathrm{NEt}_{3}$. (iii) Propargyl alcohol, $\mathrm{Pd}\left(\mathrm{PPh}_{3}\right)_{2} \mathrm{Cl}_{2}, \mathrm{CuI}, 3: 1$ $\mathrm{THF} / \mathrm{NEt}_{3}$. (iv) $\mathrm{MsCl}, \mathrm{NEt}_{3}, \mathrm{DCM}$. (v) MeOMs, MeCN. (vi) $\mathrm{MeCN}, 60^{\circ} \mathrm{C}$.

The absorbance and emission spectra of dyes $\mathbf{1}$ and $\mathbf{2}$ in chloroform are presented in Figure 1 and their photophysical

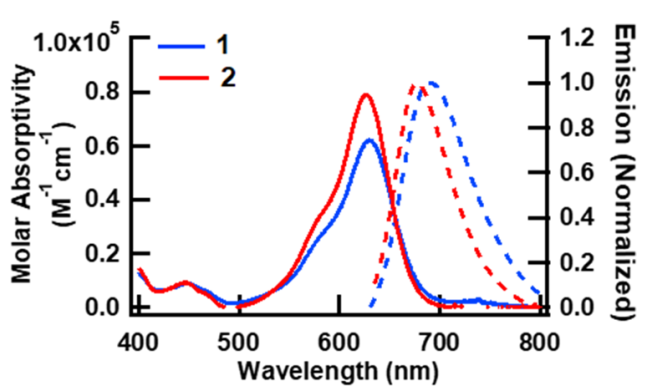

Figure 1. Absorbance (solid lines) and emission (dashed lines) spectra of dye $\mathbf{1}$ (red) and dye $\mathbf{2}$ (blue) in organic solvent.

Table 1. Photophysical Properties of 1 and 2 in $\mathrm{CHCl}_{3}$

$\begin{array}{ccccc}\text { dye } & \lambda_{\max }(\mathrm{abs}), \mathrm{nm} & \log (\varepsilon) & \lambda_{\max }(\mathrm{em}), \mathrm{nm} & \Phi_{\mathrm{F}}, \%^{a} \\ \mathbf{1} & 630 & 4.80 & 690 & 55.4 \\ \mathbf{2} & 625 & 4.90 & 678 & 51.0\end{array}$

${ }^{a}$-oxazine standard using ethanol as solvent.

properties are summarized in Table 1. Each dye shows one main absorbance band with maxima at $625-630 \mathrm{~nm}$ and one main emission band with maxima at 675-690 nm. While the shapes of the spectra are similar to other known BODIPY dyes, the maxima appear at longer wavelength. This is consistent with the extended pi electronic structures of 1 and $\mathbf{2}$ compared to other BODIPYs. Dye 1 has a slightly larger Stokes' shift (60 
$\mathrm{nm}, 0.171 \mathrm{eV})$ than dye $2(53 \mathrm{~nm}, 0.155 \mathrm{eV})$, indicating that in chloroform, the excited state of dye $\mathbf{1}$ is stabilized relative to that of dye 2; this may be due to the itaconic acid group being more inductive than the $\mathrm{DABCO}$ group. The measured molar absorptivity and fluorescence quantum yields are similar to other reported far-red BODIPYs. ${ }^{18}$

To illustrate the suitability of $\mathbf{1}$ as a fluorescent plasma membrane-specific label, we measured the absorbance and emission of 1 in water and in $0.7 \%$ Triton X-100 surfactant. The spectra and photographs of the solutions are shown in Figure 2. In pure water, the main absorbance band is broad and
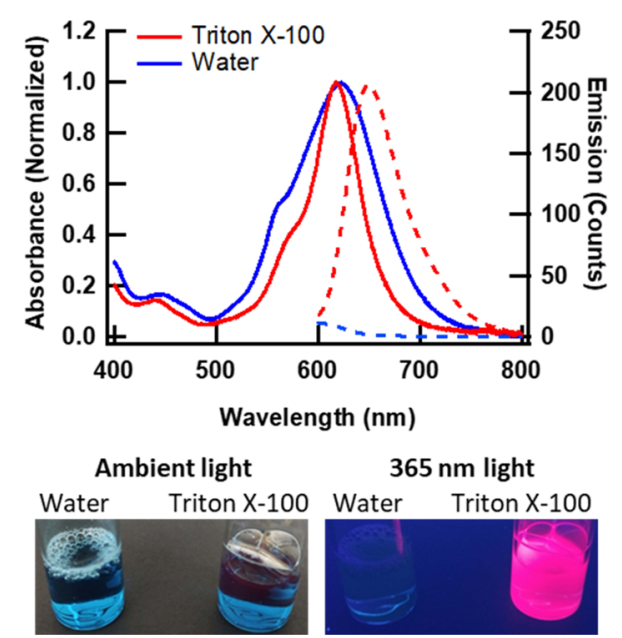

Figure 2. Top: Absorbance (solid lines) and emission (dashed lines) of 1 in water and $0.7 \%$ Triton X-100 surfactant. Bottom: photographs of 1 under ambient light (left) and $365 \mathrm{~nm}$ light (right).

featureless except for a small shoulder on the high-energy edge, and emission is minimal. However, the fluorescence excitation spectrum does not overlap with the absorbance spectrum (Supporting Information Figure S12), indicating that the detected emission is not from $\mathbf{1}$. We attribute this residual emission to either a small impurity or a slightly emissive aggregate. In Triton X-100, the main absorbance band is narrower with a more defined high-energy shoulder, and strong emission is observed. The change in absorbance in response to surfactant addition is characteristic of aggregate disruption. We believe that in pure water, the dye molecules assemble into particles with the charged groups on the surface solvated by water and the hydrophobic chromophores grouped together on the interior. Such arrangements are known to broaden absorbance curves and quench emission. Triton X-100 disrupts the aggregation by providing a hydrophobic pocket for individual dye molecules, shielding from both the water and other dye molecules, resulting in absorbance and emission characteristics similar to those observed in organic solvent. The strong fluorescence of the dyes in the surfactant solution is most likely due to both this disruption of dye aggregation and the placement of the dye core in the nonpolar environment of the micelle interior. Interestingly, 1 remains nonemissive in sodium dodecyl sulfate solution (Supporting Information Figure S13). This selectivity indicates a preference for certain matrices, highlighting its ability to be an effective membrane probe.

When applied in vivo to cells and tissues, ${ }^{34}$ the dyes similarly orient themselves with the hydrophobic chromophore embedded into the outer leaflet of the plasma membrane while the charged groups (dication for $\mathbf{1}$ and dianion for 2) remain in the extracellular space. ${ }^{35}$ It is common for dyes, even charged ones, to appear in intracellular membranes. This yields a signal that is difficult to interpret; indeed, dyes are sometimes described as dual-use with the first being a short-lived outer leaflet reporter and the second being a mitochondrial reporter. $^{36,37}$ Dianionic BODIPY 2 behaved in this manner, as shown by the diffuse intracellular emission seen in Figure 3.

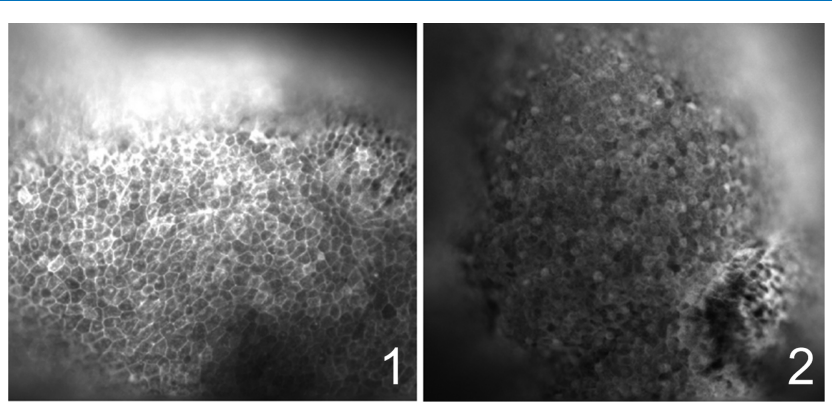

Figure 3. Fluorescence microscopy image of dye $\mathbf{1}$ (left) and dye $\mathbf{2}$ (right) labeled Xenopus tadpoles, each at $500 \mathrm{nM}$ dye concentration stained for $1 \mathrm{~h}$. Note the discrete outlining of cells for dye 1 and the diffuse staining observed with dye 2 .

This internalization is likely the result of membrane turnover beginning with endocytosis of plasma membrane. In contrast, the majority of the dicationic BODIPY 1 signal persisted in the outer leaflet for over $8 \mathrm{~h}$ of observation, with only a negligible amount entering the intracellular space (unpublished results). We believe that the presence of two charges in close proximity blocks the translocation and internalization of the dye. This allows for greatly prolonged experiments when combined with the high photostability of the dye. Furthermore, we observed no cytotoxicity with these probes at concentrations $10 \times$ the working concentration (Supporting Information Figure S14). The nonemissive nature of $\mathbf{1}$ in sodium dodecyl sulfate further indicates that $\mathbf{1}$ is only emissive in specific systems.

\section{CONCLUSIONS}

In conclusion, two new water-soluble, far-red-absorbing and NIR-emitting fluorescent dyes were synthesized that exhibit interesting amphiphilic properties. Building from a common BODIPY starting dye, dicationic and dianionic versions were made. Both of these water-soluble dyes exhibit excellent photostability and high-fluorescence quantum yields upon transition from a purely aqueous environment to one containing lipophilic regions (such as aqueous suspensions of micelles or membranes), making them intriguing candidates for cell labeling applications. Investigation of their utility as cell membrane stains and functional reporters of membrane potential changes (voltage sensitive dyes) is ongoing and will be reported in another publication.

\section{ASSOCIATED CONTENT}

\section{S Supporting Information}

The Supporting Information is available free of charge on the ACS Publications website at DOI: 10.1021/acsomega.8b01487.

Detailed experimental procedures and characterization of compounds 1-6 (PDF) 


\section{AUTHOR INFORMATION}

\section{Corresponding Author}

*E-mail: scott.selfridge@akitainnovations.com.

ORCID $\odot$

Robert Pawle: 0000-0001-9296-3928

\section{Author Contributions}

The manuscript was written through contributions of all authors.

\section{Notes}

The authors declare the following competing financial interest(s): Compounds 1 and 2 are the intellectual property of Akita Innovations, LLC, and are commercially available from Akita Innovations.

\section{ACKNOWLEDGMENTS}

Support for this work was provided by the Defense Advanced Research Projects Agency (D15PC00054) and the National Institute of Child Health and Human Development (R01HD081326). The authors thank Matthew Feeney and David Wilbur of Tufts University for their assistance with acquisition of ${ }^{13} \mathrm{C}$ NMR spectra and also thank Cheryl Wilkinson of Merrimack College for her assistance with acquisition of mass spectra.

\section{REFERENCES}

(1) Weissleder, R.; Pittet, M. J. Imaging in the era of molecular oncology. Nature 2008, 452, 580.

(2) The Molecular Probes Handbook: A Guide to Fluorescent Probes and Labelling Technologies, 11th ed.; ThermoFisher Scientific: Carlsbad, CA, 2010.

(3) Yi, X. M.; Wang, F. L.; Quin, W. J.; Yang, X. J.; Yuan, J. L. NearInfrared Fluorescent Prodes in Cancer Imaging and Therapy: an Emerging Field. Int. J. Nanomed. 2014, 9, 1347-1365.

(4) Devaraj, N. K.; Weissleder, R.; Hilderbrand, S. A. TetrazineBased Cycloadditions: Application to Pretargeted Live Cell Imaging. Bioconjugate Chem. 2008, 19, 2297-2299.

(5) Ziessel, R.; Ulrich, G.; Harriman, A.; Alamiry, M. A. H.; Stewart, B.; Retailleau, P. Solid-State Gas Sensors Developed from Functional Difluoroboradiazaindacene Dyes. Chem. - Eur. J. 2009, 15, 13591369

(6) Moriarty, R. D.; Martin, A.; Adamson, K.; O’Reilly, E.; Mollard, P.; Forster, R. J.; Keyes, T. E. The application of water soluble, megaStokes-shifted BODIPY fluorophores to cell and tissue imaging. $J$. Microsc. 2014, 253, 204-218.

(7) Mula, S.; Ray, A. K.; Banerjee, M.; Chaudhuri, T.; Dasgupta, K.; Chattopadhyay, S. Design and Development of a New Pyrromethene Dye with Improved Photostability and Lasing Efficiency: Theoretical Rationalization of Photophysical and Photochemical Properties. J. Org. Chem. 2008, 73, 2146-2154.

(8) Lavis, L. D.; Raines, R. T. Bright Ideas for Chemical Biology. ACS Chem. Biol. 2008, 3, 142-155.

(9) Ni, Y.; Zeng, L.; Kang, N. Y.; Huang, K. W.; Wang, L.; Zeng, Z.; Chang, Y. T.; Wu, J. meso-Ester and Carboxylic Acid Substituted BODIPYs with Far-Red and Near-Infrared Emission for Bioimaging Applications. Chem. - Eur. J. 2014, 20, 2301-2310.

(10) Escobedo, J. O.; Rusin, O.; Lim, S.; Strongin, R. M. NIR dyes for bioimaging applications. Curr. Opin. Chem. Biol. 2010, 14, 64-70.

(11) Mishra, A.; Behera, R. K.; Behera, P. K.; Mishra, B. K.; Behera, G. B. Cyanines during the 1990s: A Review. Chem. Rev. 2000, 100, 1973-2012.

(12) Berezin, M. Y.; Achilefu, S. Fluorescence Lifetime Measurements and Biological Imaging. Chem. Rev. 2010, 110, 2641-2684.

(13) Drummen, G. P. Fluorescent Probes and Fluorescence (Microscopy) Techniques - Illuminating Biological and Biomedical Research. Molecules 2012, 17, 14067.
(14) Ajayaghosh, A. Chemistry of Squaraine-Derived Materials: Near-IR Dyes, Low Band Gap Systems, and Cation Sensors. Acc. Chem. Res. 2005, 38, 449-459.

(15) Bioconjugate Techniques, 2nd ed.; Hermanson, G. T., Ed.; Academic Press: New York, 2008.

(16) Jose, J.; Burgess, K. Syntheses and Properties of Water-Soluble Nile Red Derivatives. J. Org. Chem. 2006, 71, 7835-7839.

(17) Treibs, A.; Kreuzer, F. H. Difluorboryl-Komplexe von Di- und Tripyrrylmethenen. Justus Liebigs Ann. Chem. 1968, 718, 208-223.

(18) Loudet, A.; Burgess, K. BODIPY Dyes and Their Derivatives: Syntheses and Spectroscopic Properties. Chem. Rev. 2007, 107, 48914932.

(19) Ulrich, G.; Ziessel, R.; Harriman, A. The Chemistry of Fluorescent Bodipy Dyes: Versatility Unsurpassed. Angew. Chem., Int. Ed. 2008, 47, 1184-1201.

(20) Niu, S. L.; Ulrich, G.; Ziessel, R.; Kiss, A.; Renard, P.-Y.; Romieu, A. Water-Soluble BODIPY Derivatives. Org. Lett. 2009, 11, 2049-2052.

(21) Ni, Y.; Wu, J. Far-red and near infrared BODIPY dyes: synthesis and applications for fluorescent $\mathrm{pH}$ probes and bio-imaging. Org. Biomol. Chem. 2014, 12, 3774-3791.

(22) Alamudi, S. H.; Su, D.; Lee, K. J.; Lee, J. Y.; Belmonte-Vázquez, J. L.; Park, H.-S.; Peña-Cabrera, E.; Chang, Y.-T. A palette of background-free tame fluorescent probes for intracellular multi-color labelling in live cells. Chem. Sci. 2018, 9, 2376-2383.

(23) Chen, H.; He, X.; Su, M.; Zhai, W.; Zhang, H.; Li, C. A General Strategy Toward Highly Fluorogenic Bioprobes Emitting across the Visible Spectrum. J. Am. Chem. Soc. 2017, 139, 10157-10163.

(24) Miao, F.; Uchinomiya, S.; Ni, Y.; Chang, Y.-T.; Wu, J. Development of $\mathrm{pH}$-Responsive BODIPY Probes for Staining Late Endosome in Live Cells. ChemPlusChem 2016, 81, 1209-1215.

(25) Jia, H.-R.; Wang, H.-Y.; Yu, Z.-W.; Chen, Z.; Wu, F.-G. LongTime Plasma Membrane Imaging Based on a Two-Step Synergistic Cell Surface Modification Strategy. Bioconjugate Chem. 2016, 27, $782-789$.

(26) Wang, H.-Y.; Hua, X.-W.; Jia, H.-R.; Li, C.; Lin, F.; Chen, Z.; Wu, F.-G. Universal Cell Surface Imaging for Mammalian, Fungal, and Bacterial Cells. ACS Biomater. Sci. Eng. 2016, 2, 987-997.

(27) Chen, X.; Zhang, X.; Wang, H.-Y.; Chen, Z.; Wu, F.-G. Subcellular Fate of a Fluorescent Cholesterol-Poly(ethylene glycol) Conjugate: An Excellent Plasma Membrane Imaging Reagent. Langmuir 2016, 32, 10126-10135.

(28) Takahashi, D.; Koda, Y.; Sasaki, Y.; Akiyoshi, K. Design and synthesis of PEGylated amphiphilic block oligomers as membrane anchors for stable binding to lipid bilayer membranes. Polym. J. 2018, 50, 787-797.

(29) Tahtaoui, C.; Thomas, C.; Rohmer, F.; Klotz, P.; Duportail, G.; Mély, Y.; Bonnet, D.; Hibert, M. Convenient Method To Access New 4,4-Dialkoxy- and 4,4-Diaryloxy-diaza-s-indacene Dyes: Synthesis and Spectroscopic Evaluation. J. Org. Chem. 2007, 72, 269-272.

(30) Burghart, A.; Kim, H.; Welch, M. B.; Thoresen, L. H.; Reibenspies, J.; Burgess, K.; Bergström, F.; Johansson, L. B. Å. 3,5Diaryl-4,4-difluoro-4-bora-3a,4a-diaza-s-indacene (BODIPY) Dyes: Synthesis, Spectroscopic, Electrochemical, and Structural Properties. J. Org. Chem. 1999, 64, 7813-7819.

(31) Dost, Z.; Atilgan, S.; Akkaya, E. U. Distyryl-boradiazaindacenes: facile synthesis of novel near IR emitting fluorophores. Tetrahedron 2006, 62, 8484-8488.

(32) Rurack, K.; Kollmannsberger, M.; Daub, J. Molecular Switching in the Near Infrared (NIR) with a Functionalized BoronDipyrromethene Dye. Angew. Chem., Int. Ed. 2001, 40, 385-387.

(33) Yu, Y. H.; Descalzo, A. B.; Shen, Z.; Röhr, H.; Liu, Q.; Wang, Y. W.; Spieles, M.; Li, Y. Z.; Rurack, K.; You, X. Z. Mono- and Di(dimethylamino)styryl-Substituted Borondipyrromethene and Borondiindomethene Dyes with Intense Near-Infrared Fluorescence. Chem. - Asian J. 2006, 1, 176-187.

(34) Devaraj, N. K.; Hilderbrand, S.; Upadhyay, R.; Mazitschek, R.; Weissleder, R. Bioorthogonal Turn-On Probes for Imaging Small 
Molecules inside Living Cells. Angew. Chem., Int. Ed. 2010, 49, 28692872.

(35) Dahim, M.; Mizuno, N. K.; Li, X.-M.; Momsen, W. E.; Momsen, M. M.; Brockman, H. L. Physical and Photophysical Characterization of a BODIPY Phosphatidylcholine as a Membrane Probe. Biophys. J. 2002, 83, 1511-1524.

(36) Zhang, X.; Xiao, Y.; Qi, J.; Qu, J.; Kim, B.; Yue, X.; Belfield, K. D. Long-Wavelength, Photostable, Two-Photon Excitable BODIPY Fluorophores Readily Modifiable for Molecular Probes. J. Org. Chem. 2013, 78, 9153-9160.

(37) Farkas, D. L.; Wei, M. D.; Febbroriello, P.; Carson, J. H.; Loew, L. M. Simultaneous imaging of cell and mitochondrial membrane potentials. Biophys. J. 1989, 56, 1053-1069. 